\title{
The role of IncRNA MALAT1 in the regulation of hepatocyte proliferation during liver regeneration
}

\author{
CUICUI LI ${ }^{1 *}$, LEI CHANG ${ }^{2 *}$, ZHIQUAN CHEN $^{1}$, ZHONGZHONG LIU $^{1}$, YANFENG WANG $^{1}$ and QIFA YE $^{1}$ \\ ${ }^{1}$ Zhongnan Hospital of Wuhan University, Institute of Hepatobiliary Diseases of Wuhan University, Transplant Center of \\ Wuhan University, Hubei Key Laboratory of Medical Technology on Transplantation; ${ }^{2}$ Department of General Surgery, \\ Zhongnan Hospital of Wuhan University, Wuhan, Hubei 430071, P.R. China
}

Received May 22, 2016; Accepted December 29, 2016

DOI: $10.3892 /$ ijmm.2017.2854

\begin{abstract}
Exploring the biological functions of long noncoding RNAs (lncRNAs) has come to the foreground in recent years. Studies have indicated that the IncRNA metastasis-associated lung adenocarcinoma transcript 1 (MALAT1) not only regulates tumorigenesis in hepatocellular carcinoma, but also controls cell cycle progression in hematopoietic cells. The present study was designed to investigate the biological role of lncRNA MALAT1 in liver regeneration. We carried out a series of assays during liver regeneration following $2 / 3$ partial hepatectomy in mice. We explored the functions of lncRNA MALAT1 with a series of functional analyses in vitro. We found that MALAT1 was upregulated during liver regeneration. Moreover, MALAT1 accelerated hepatocyte proliferation by stimulating cell cycle progression from the $\mathrm{G} 1$ to the $\mathrm{S}$ phase and inhibiting apoptosis in vitro. In addition, our findings also demonstrated that MALAT1 was regulated by p53 during liver regeneration, and that p53 may be a key upstream regulator of MALAT1 activity. Mechanistically, we found that MALAT1 activated the Wnt/ $\beta$-catenin pathway by inhibiting the expression of Axin1 and adenomatous polyposis coli (APC), and subsequently promoting the expression of cyclin D1. On the whole, the findings of this study suggest that MALAT1 is a critical molecule for liver regeneration. Pharmacological interventions targeting MALAT1 may thus prove to be therapeutically beneficial in liver failure or liver transplantation by promoting liver regeneration.
\end{abstract}

Correspondence to: Dr Qifa Ye, Zhongnan Hospital of Wuhan University, Institute of Hepatobiliary Diseases of Wuhan University, Transplant Center of Wuhan University, Hubei Key Laboratory of Medical Technology on Transplantation, 169 Donghu Road, Wuhan, Hubei 430071, P.R. China

E-mail: yqf_china@163.com

*Contributed equally

Key words: long non-coding RNA, metastasis-associated lung adenocarcinoma transcript 1 , liver regeneration, 2/3 partial hepatectomy, Wnt//3-catenin, p53

\section{Introduction}

Liver regeneration refers to the liver repair process that occurs following the loss of part of the liver due to surgery, trauma, poisoning, infection and necrosis, or liver transplantation. When liver regeneration occurs, hepatocytes in the G0 phase are stimulated by various feedback signals to enter a period of rapid growth. Liver regeneration has valuable implications for hepatobiliary surgeries, such as the resection of a liver mass, liver transplantation and split liver transplantation, as the above-mentioned surgical procedures depend largely on the ability of the liver to regenerate. Poor liver regeneration may lead to the death of the patient, for example, via smallfor-size syndrome (SFSS) (1-4). For that reason, understanding the mechanisms of liver regeneration has great implications for clinical practice. A series of interacting factors, including inflammatory cytokines (5), hepatocyte growth factor (HGF) (6), cell cycle regulators, cyclin-dependent kinases and metabolic regulators control the hepatocyte cell cycle to ensure the rapid and orderly progression of liver regeneration (7). Studies have indicated that microRNAs (miRNAs or miRs) play a key role in liver regeneration (8-10). Despite the identification of many cytokines, miRNAs and growth factors that regulate the expression of genes controlling proliferation during liver regeneration (11), the exact molecular mechanisms which are responsible for liver regeneration remain unclear, thus prompting our interest to investigate the molecular mechanisms underlying liver regeneration.

In recent years, the field of RNA biology has been dominated by the study of miRNAs; however, several major biological processes are also regulated by long non-coding RNAs (lncRNAs), including carcinogenesis, development and differentiation $(12,13)$. IncRNA molecules are $>200$ nucleotides in length and do not encode proteins. Studies have reported that some lncRNAs, including lncRNA H19 and lncRNA LALR1, play key roles during liver regeneration $(14,15)$. IncRNA H19 promotes hepatocyte proliferation during liver regeneration in both the rat and mouse (15). Improving the effectiveness of current regeneration techniques may help to identify a potential therapeutic target to stimulate liver regeneration.

Metastasis-associated lung adenocarcinoma transcript 1 (MALAT1) is one of the most widely studied lncRNAs and is frequently overexpressed in malignant tumors; therefore, 
MALAT1 is frequently regarded as a biomarker for a variety of tumors. A previous study demonstrated that MALAT1 localizes in dynamic structures known as nuclear speckles, which are key elements in the collection and recruitment of splicing factors (16). Researchers have also found that MALAT1 plays a variety of biological functions in hematopoietic cells, including the regulation of the cell cycle (17). However, the biological role of the IncRNA MALAT1 in liver regeneration and its mechanisms of action regarding the regulation of hepatocyte proliferation remain unclear.

In this study, we focused on the mechanisms underlying the MALAT1-mediated modulation of hepatocyte proliferation. We investigated the role of MALAT1 in the regulation of liver regeneration with a murine model of $2 / 3$ partial hepatectomy $(\mathrm{PH})$ and an in vitro hepatocyte proliferation assay. The comprehensive dynamic changes in MALAT1 expression were examined at multiple time points (including 0, 2, $6,12,15,18,24,36,48,72,120,144$ and $168 \mathrm{~h}$ ) following 2/3 PH; we then aimed to elucidate the mechanisms through which MALAT1 regulates liver regeneration in the mouse. We found that MALAT1 promoted hepatocyte proliferation and increased the expression of cyclin D1 by activating the $\mathrm{Wnt} / \beta$-catenin pathway. We also found that the relative expression level of $\mathrm{p} 53$, which is a negative regulator of MALAT1, was the opposite of MALAT1 expression following 2/3 PH. Our study may have identified a potential therapeutic target for use during liver regeneration.

\section{Materials and methods}

Animals and model of $2 / 3 \mathrm{PH}$. A total of $60 \mathrm{male} \mathrm{BALB} / \mathrm{c}$ mice (8-12 weeks old, weighing $23 \pm 2 \mathrm{~g}$ ) were purchased from the Central Laboratory of Animal Science, Wuhan University (Wuhan, China) and were maintained in a specific pathogenfree facility with a 12-h light/dark cycle and were fed standard laboratory chow with free access to water ad arbitrium. All the animal protocols used in the study were reviewed and approved by the Animal Experiment Center of Wuhan University/Animal Biosafety Level-III Laboratory. 2/3 PH was performed on 8-12-week-old mice using a previously described method (18). Following a laparotomy, the left and median liver lobes were resected in two steps. Five mice were used for each $2 / 3 \mathrm{PH}$ time point $(0,2,6,12,15,18,24,36,48$, 72,120 and $168 \mathrm{~h}$ ). The end of surgery was considered $0 \mathrm{~h}$. All the partial hepatectomies were performed in the early morning hours. The mice were sacrificed (by cervical dislocation) at defined time points following PH. The mice were sacrificed under isoflurane anesthesia as previously described (18). The remaining parts of the livers were surgically removed, cleaned with saline solution and were snap-frozen in liquid nitrogen for RNA and protein analysis and kept at $-80^{\circ} \mathrm{C}$ until further use or fixed in $4 \%$ paraformaldehyde for histological analysis and processed for immunohistochemistry. For histological analysis, after being fixed in $4 \%$ paraformaldehyde for $48 \mathrm{~h}$, the tissues were embedded in paraffin. The total body weight was measured, and the remnant and regenerated liver tissues were resected and weighed. The acquired data were expressed as a percentage of the ratio between the remnant liver weight (A) divided by the total body weight (B) x100 (liver-to-body weight ratio $(\%)=\mathrm{A} / \mathrm{B} \times 100)$.
Table I. Primer and target sequences used in this study.

\begin{tabular}{|c|c|}
\hline Genes & Sequences or target sequences $\left(5^{\prime} \rightarrow^{\prime}\right)$ \\
\hline MALAT1-F & TGCAGTGTGCCAATGTTTCG \\
\hline MALAT1-R & GGCCAGCTGCAAACATTCAA \\
\hline GAPDH-F & GGTGAAGGTCGGTGTGAACG \\
\hline GAPDH-R & СTCGCTCCTGGAAGATGGTG \\
\hline HGF-F & TCATTGGTAAAGGAGGCA \\
\hline HGF-R & GTCACAGACTTCGTAGCG \\
\hline$\beta$-catenin-F & ATGGCTTGGAATGAGACT \\
\hline$\beta$-catenin-R & TGAGGTCCTGGGCGTGT \\
\hline Cyclin D1-F & GAGGAGCAGAAGTGCGAAGA \\
\hline Cyclin D1-R & GCCGGATAGAGTTGTCAGTGTAC \\
\hline Axin1-F & GCTGCTATTGGAGACTGCT \\
\hline Axin1-FR & GTACCCGCCCATTGACT \\
\hline GSK-3 $\beta-F$ & TTATTTGACCGCATAGTTC \\
\hline GSK-3 $\beta-R$ & AAGCACCTGACTTTCCTC \\
\hline APC-F & CCTGTGGCAAGGAAACC \\
\hline APC-R & CTCGCTGAGCATCATCTGT \\
\hline p53-F & CAGCCCCCTCTCTGAGTAGT' \\
\hline $\mathrm{p} 53-\mathrm{R}$ & ACCCTATGAGGGCCCAAGAT \\
\hline \multirow[t]{2}{*}{ MALAT1-siRNA-1 } & GCUCAGGACUUUGCAUAUATT \\
\hline & UAUAUGCAAAGUCCUGAGCTT \\
\hline \multirow[t]{2}{*}{ MALAT1-siRNA-2 } & GCAGAAGAGUUGCUUCAUUTT \\
\hline & AAUGAAGCAACUCUUCUGCTT \\
\hline \multirow[t]{2}{*}{ MALAT1-siRNA-3 } & GCGGAAUUGCUGGUAGUUUTT \\
\hline & AAACUACCAGCAAUUCCGCTT \\
\hline \multirow{2}{*}{$\begin{array}{l}\text { MALAT1-siRNA- } \\
\text { scramble }\end{array}$} & UUCUCCGAACGUGUCACGUTT \\
\hline & ACGUGACACGUUCGGAGATTT \\
\hline
\end{tabular}

MALAT1, metastasis-associated lung adenocarcinoma transcript 1; GAPDH, glyceraldehyde 3-phosphate dehydrogenase; HGF, hepatocyte growth factor; APC, adenomatous polyposis coli.

Histological analysis. At different time points following $2 / 3 \mathrm{PH}$, the animals were sacrificed and the liver tissues were harvested. Liver tissues were obtained from the remnant livers and then fixed immediately in $10 \%$ buffered formalin phosphate solution, embedded in paraffin and were then cut into 5-mm-thick sections. The sections were stained with hematoxylin and eosin (H\&E). The H\&E staining kit was obtained from Service Bio Co., Ltd. (Wuhan, China Cat. no. G1005). All tissue sections were examined with an Olympus BH-2 microscope (Olympus Optical Co. Ltd., Beijing, China). Motic Images 2000 (Motic China Group Co. Ltd, Guangzhou, China) was used for the characterization of histopathological changes.

ELISA. The mice were sacrificed and blood samples were collected via the post-orbital venous plexus at the mentioned observation time points. Serum samples were separated and stored at $-80^{\circ} \mathrm{C}$ until further use. The serum levels of alanine aminotransferase (ALT), aspartate aminotransferase (AST) and albumin (ALB) were measured using respective ELISA kits (Nanjing Jiancheng Biological Technology, Inc., Nanjing, China) as previously described (19). 
Table II. Information regarding the antibodies used in this study.

\begin{tabular}{|c|c|c|c|c|c|}
\hline Antibody & WB & $\mathrm{IHC}$ & $\mathrm{IF}$ & Specificity & Company \\
\hline GAPDH (10494-1-AP) & $1: 5,000$ & & & Rabbit polyclonal & Proteintech \\
\hline$\beta$-catenin (no. 8480) & $1: 1,000$ & & $1: 100$ & Rabbit monoclonal & Cell Signaling Technology \\
\hline Cyclin D1 (no. 2978) & $1: 1,000$ & & & Rabbit monoclonal & Cell Signaling Technology \\
\hline Axin1 (no. 2087) & $1: 1,000$ & & & Rabbit monoclonal & Cell Signaling Technology \\
\hline c-Myc (sc-788) & $1: 200$ & & & Rabbit polyclonal & Santa Cruz Biotechnology \\
\hline Cyclin B1 (55004-1-AP) & $1: 2,000$ & & & Rabbit polyclonal & Proteintech \\
\hline Cyclin E1 (11554-1-AP) & $1: 1,000$ & & & Rabbit polyclonal & Proteintech \\
\hline GSK-3 $\beta$ (no. 12456) & $1: 1,000$ & & & Rabbit polyclonal & Cell Signaling Technology \\
\hline APC (sc-896) & $1: 200$ & & & Rabbit polyclonal & Santa Cruz Biotechnology \\
\hline p53 (sc-6243) & $1: 200$ & $1: 200$ & & Rabbit polyclonal & Santa Cruz Biotechnology \\
\hline PCNA (10205-2-AP) & $1: 2,000$ & $1: 200$ & & Rabbit polyclonal & Proteintech \\
\hline Histone H3 (no. 4499) & $1: 2,000$ & & & Rabbit monoclonal & Cell Signaling Technology \\
\hline
\end{tabular}

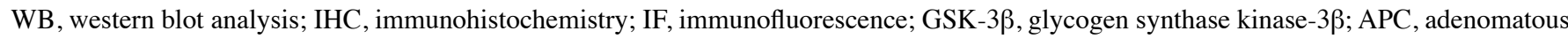
polyposis coli; PCNA, proliferating cell nuclear antigen.

Reverse transcription-quantitative PCR (RT-qPCR). Total RNA was isolated from the frozen tissues or cells using TRIzol reagent (Invitrogen Life Technologies, Carlsbad, CA, USA) according to the manufacturer's instructions. For RT-qPCR, a reverse transcription kit (Takara Biotechnology Co., Ltd. Dalian, China) was used to synthesize cDNA. A SYBRGreen PCR kit (Toyobo, Osaka, Japan) was used to complete the RT-qPCR analyses. PCR reactions were performed on a CFX96 system (Bio-Rad CFX96; Bio-Rad Laboratories, Inc., Hercules, CA, USA). The results of RT-qPCR were normalized to glyceraldehyde 3-phosphate dehydrogenase (GAPDH), and the $2^{-\triangle \Delta C T}$ values were normalized to the GAPDH levels. All the RT-qPCR experiments were repeated at least 3 times. The primer sequences used in this study are listed in Table I.

Cell culture and transfection. NCTC 1469 and BNL CL.2 cells were obtained from the China Center for Type Culture Collection (Wuhan, China). The NCTC 1469 cells were cultured with Dulbecco's modified Eagle's medium (DMEM H-21, $4.5 \mathrm{~g} / 1$ glucose) supplemented with $15 \%$ horse serum. BNL CL. 2 cells were cultured with DMEM supplemented with $10 \%$ fetal bovine serum (FBS). The growth media used in the present study contained $5 \mathrm{U} / \mathrm{ml}$ penicillin and $5 \mu \mathrm{g} / \mathrm{ml}$ streptomycin sulfate. Full-length MALAT1 (GenBank: NR_002847.2) was cloned into the pcDNA3.1 vector (Invitrogen Life Technologies). An empty vector (pcDNA3.1) was used as a control. siRNAs against MALAT1 were designed and synthesized by Beijing View Solid Biotechnology (Beijing, China). The siRNA target sequences used in this study are listed in Table I. The MALAT1 expression levels were detected by RT-qPCR. Scramble siRNA was used as a control. Transfection was conducted using a LipoJet ${ }^{\mathrm{TM}}$ in vitro DNA and siRNA transfection kit (both from SignaGen Laboratories, Gaithersburg, MD, USA) according to the manufacturer's instructions. The hepatocytes were fixed with $4 \%$ paraformaldehyde $48 \mathrm{~h}$ after transfection for further analysis.

NCTC 1469 cells were treated with HGF (Cat. no. \#315-23; PeproTech, Rocky Hill, NJ, USA) using a concentration range of $5-20 \mathrm{ng} / \mathrm{ml}$. It is recommended to reconstitute the lophilized $\mathrm{HGF}$ in sterile $18 \mathrm{M} \Omega-\mathrm{cm} \mathrm{H}_{2} \mathrm{O}$ not less than $100 \mu \mathrm{g} / \mathrm{ml}$, which can then be further diluted to other aqueous solutions.

Flow cytometric analysis. The cells transfected with siRNA or the pcDNA3.1 vector were collected at $48 \mathrm{~h}$ post-transfection. Following double-labeling with Annexin V-FITC and propidium iodide (PI), apoptosis was analyzed using a flow cytometer (FACScan ${ }^{\circledR}$; BD Biosciences, Franklin Lakes, NJ, USA) equipped with CellQuest 3.3 software. For the cell cycle analysis, $1 \times 10^{6}$ cells were plated into a $60-\mathrm{mm}$ dish and incubated for $24 \mathrm{~h}$. The cells were subsequently stained with $1 \mathrm{ml}$ of staining solution [PI $(50 \mu \mathrm{g} / \mathrm{ml})$ and RNase A $(20 \mu \mathrm{g} /$ $\mathrm{ml})$ ] for 15 min using the Cycletest ${ }^{\mathrm{TM}}$ PLUS DNA reagent kit (BD Biosciences) according to the manufacturer's instructions.

Western blot analysis. The liver tissue samples and hepatocytes were homogenized in lysis buffer (Beyotime Institute of Biotechnology, Haimen, China) and incubated for $30 \mathrm{~min}$ on ice. For the detection of $\beta$-catenin, cytoplasmic and nuclear protein extracts were prepared from the cells using the NE-PER Nuclear and Cytoplasmic Extraction Reagent kit (Cat. no. 78835; Thermo Fisher Scientific, Inc., Waltham, MA, USA) according to the manufacturer's instructions. Total protein was electrophoresed by sodium dodecyl sulfate-polyacrylamide gel electrophoresis (SDS-PAGE) and then transferred onto polyvinylidene fluoride (PVDF) membranes (Millipore, Billerica, MA, USA). The membranes were incubated with the corresponding primary antibodies overnight at $4^{\circ} \mathrm{C}$. The following day, the membranes were incubated with secondary antibodies [goat anti-rabbit IgG (H+L)-HRP (LK2001; Sungene Biotech Co., Ltd., Tianjin, China)] for 1-2 h. Proteins were detected using the ECL immunoblotting kit (Beyotime Institute of Biotechnology). The fluorescent bands were observed in a dark room with infrared light. The above procedure was performed according to the manufacturer's instructions. The information for the antibodies is listed in Table II. 
Cell proliferation assay. A Cell Counting Kit-8 kit (CCK-8; Dojindo, Kumamoto, Japan) was used for the cell proliferation assays. Approximately $3-5 \times 10^{4}$ cells were plated in triplicate in 96-well plates and cultured in growth medium. The plate was incubated at $37^{\circ} \mathrm{C}$ and $5 \% \mathrm{CO}_{2}$ for an appropriate length of time (4-6 h) until cell adherence. Ten microliters of CCK-8 solution were then added to each well of the plate. The absorbance was measured at $450 \mathrm{~nm}$ using a microplate reader $(168-1000 \mathrm{XC}$; Bio-Rad Laboratories, Inc.) at the indicated time points.

5-Ethynyl-2'-deoxyuridine (EdU) incorporation assay. The EdU incorporation assay was performed using an EdU Apollo DNA in vitro kit (C10310-1; Guangzhou RiboBio Co.,Ltd., Guangzhou, China) according to the manufacturer's instructions. The hepatocytes were stained with EdU $48 \mathrm{~h}$ following transfection. First, the cells were incubated with $100 \mathrm{ml}$ of $50 \mathrm{mM}$ EdU/well for $2 \mathrm{~h}$. The cells were then fixed with $4 \%$ paraformaldehyde for $15 \mathrm{~min}$ at room temperature. Following fixation and washing with phosphate-buffered saline (PBS), the cells were incubated with $50 \mu \mathrm{l}$ of $2 \mathrm{mg} / \mathrm{ml}$ glycine for $5 \mathrm{~min}$. One hundred microliters of permeabilization buffer (PBS containing $0.5 \%$ Triton X-100) was added to each well, and the cells were incubated with $100 \mu 1 \mathrm{1X}$ Apollo solution for $30 \mathrm{~min}$ at room temperature in the dark. Then, $100 \mu 11 \mathrm{X}$ Hoechst 33342 solution was added dropwise into each cell climbing slice for $15 \mathrm{~min}$. The cell climbing slices were washed, dried, mounted and then observed under a fluorescence microscope (Olympus BX53; Olympus, Tokyo, Japan).

Immunohistochemistry (IHC) and immunofluorescence (IF). Paraffin-embedded tissue was cut into $4-\mu \mathrm{m}$-thick sections and processed for IHC in accordance with a previously described protocol (20). For IF, the cells were fixed with $4 \%$ paraformaldehyde, permeabilized using $0.2 \%$ Triton X-100 and incubated with primary and secondary antibodies according to the manufacturer's instructions. The cells were counterstained with 4',6-diamidino-2-phenylindole (DAPI) (Calbiochem, San Diego, CA, USA) and imaged using a confocal laser scanning microscope (Olympus FV1000; Olympus).

Statistical analysis. Quantitative data are presented as the means \pm standard deviation (SD) from at least 3 independent experiments. Statistical significance was assessed using the Student's t-test, $\chi^{2}$ test or Fisher's exact test, which were performed using SPSS 22.0 statistical software (IBM SPSS, Inc., Chicago, IL, USA). Differences were considered statistically significant when P-values were $<0.05$.

\section{Results}

MALAT1 is upregulated during liver regeneration. To determine the impact of MALAT1 on liver regeneration, we first generated mouse model of $2 / 3 \mathrm{PH}$. To assess rehabilitation from acute liver injury, the serum levels of ALT, AST and ALB were determined 0, 24, 36, 48, 72 and $168 \mathrm{~h}$ following 2/3 $\mathrm{PH}$. As shown in Fig. $1 \mathrm{~A}-\mathrm{C}$, all the indicators returned to normal at $168 \mathrm{~h}$ following $2 / 3 \mathrm{PH}$. H\&E staining of the liver was carried out at different time points. Following hepatectomy, we observed obvious cell edema at 15 and $36 \mathrm{~h}$. Mitotic figures were clearly observed at $72 \mathrm{~h}$, and the hepatic lobe structure was close to normal at $120 \mathrm{~h}$ (Fig. 1D). Mouse hepatocyte proliferation was determined in vivo by staining for proliferating cell nuclear antigen (PCNA) (Fig. 1D) using IHC. We found that the number of PCNA-positive nuclei reached a peak at $36 \mathrm{~h}$ following $2 / 3 \mathrm{PH}$. The cell proliferative ability was reduced at 72 and $120 \mathrm{~h}$, and the proliferation at 24 and $168 \mathrm{~h}$ was not significant (Fig. 1D). The remnant liver/body weight ratio is shown in Fig. 1E.

2/3 $\mathrm{PH}$ in male BALB/c mice caused a $>5$-fold increase in MALAT1 expression. MALAT1 expression increased from $2 \mathrm{~h}$, peaked at $15 \mathrm{~h}$ following $\mathrm{PH}$, and then returned to almost normal levels $120 \mathrm{~h}$ following 2/3 PH (Fig. 1F). These results suggested that MALAT1 was specifically overexpressed in hepatic tissue following 2/3 PH. Taken together, this sharp increase indicates that MALAT1 may play a role in liver regeneration.

IncRNA MALAT1 accelerates cell cycle progression in hepatocytes and promotes proliferation in vitro. As it has been previously demonstrated that hepatocytes from adult male mice enter the $\mathrm{S}$ phase at $36 \mathrm{~h}$ following $2 / 3 \mathrm{PH}$ (18) and that the expression of MALAT1 reaches a maximum after $12-18 \mathrm{~h}$ of liver regeneration, we hypothesized that MALAT1 may play an important role in regulating the cell cycle, as the expression of MALAT1 peaked before $36 \mathrm{~h}$.

First, we analyzed MALAT1 expression in BNL CL.2 and NCTC 1469 cells. MALAT1 expression was significantly higher in the BNL CL. 2 cells than in the NCTC 1469 cells (Fig. 2A). As shown in Fig. 2B, siRNA-2 was the most effective at silencing MALAT1. MALAT1 expression was upregulated almost 18-fold following transfection of the NCTC 1469 cells with the pcDNA3.1 vector carrying MALAT1. CCK-8 (Fig. 2C) and EdU immunofluorescence assays (Fig. 2D) were used to detect changes in the proliferative ability of the MALAT1silenced BNL CL.2 cells and MALAT1-overexpressing NCTC 1469 cells. The experimental results revealed that cell proliferation was reduced when MALAT1 was silenced in the BNL CL. 2 cells and was increased when MALAT1 was overexpressed in the NCTC 1469 cells. As shown in Fig. 2D, the MALAT1-overexpressing NCTC 1469 cells had more EdU-positive cells than the control group, while the MALAT1-silenced BNL CL.2 cells had fewer EdU-positive cells than the control cells. Moreover, FACS analysis indicated that the MALAT1-silenced BNL CL.2 cells were arrested in the G0/G1 phase of the cell cycle. Furthermore, the number of cells in the G0/G1 phase decreased significantly and the number of cells in the $S$ phase increased significantly when MALAT1 was overexpressed in the NCTC 1469 cells (Fig. 2E). At the same time, the rate of apoptosis increased when the BNL CL.2 cells were transfected with MALAT1-siRNA-2, but decreased when the NCTC 1469 cells were transfected with pcDNA3.1-MALAT1 (Fig. 2F). To further examine the role of MALAT1 in cell cycle progression, the expression of cyclin E1 and B1 was examined in the MALAT1-silenced BNL CL.2 cells. The results revealed that the G0/G1 phase was extended when MALAT1 was silenced in the BNL CL.2 cells (Fig. 2G). Thus, the above-mentioned data indicate that MALAT1 facilitates liver cell proliferation by accelerating cell cycle progression and blocking liver cell apoptosis. 

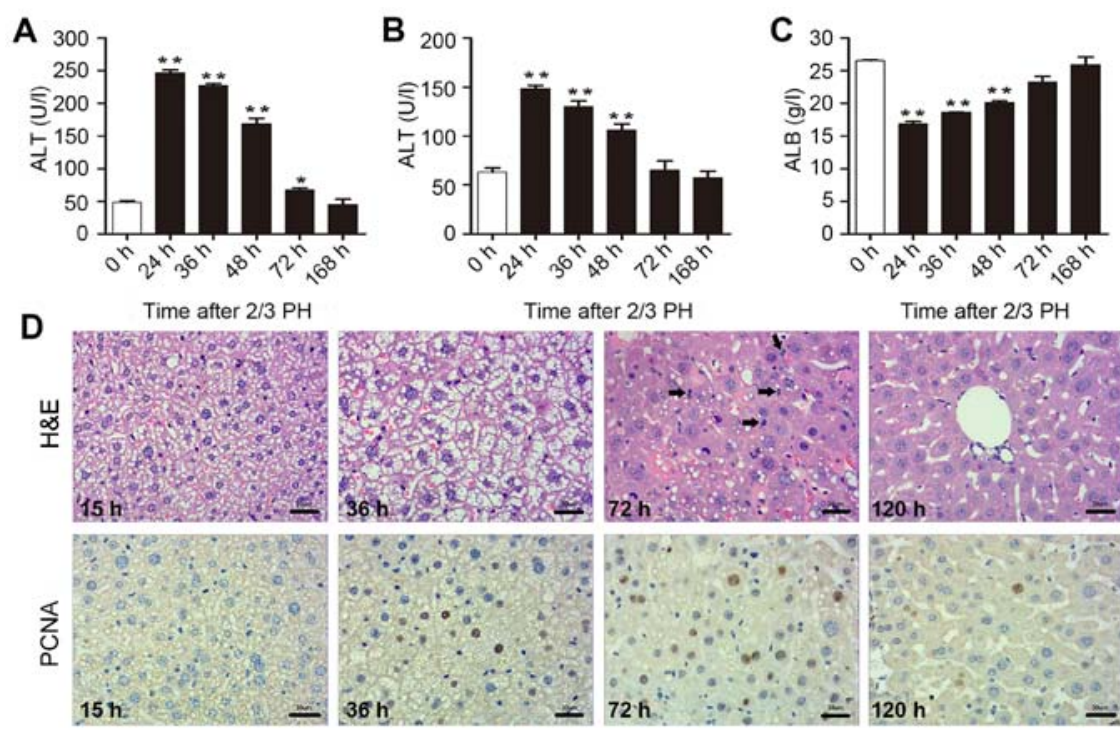

Time after $2 / 3 \mathrm{PH}$
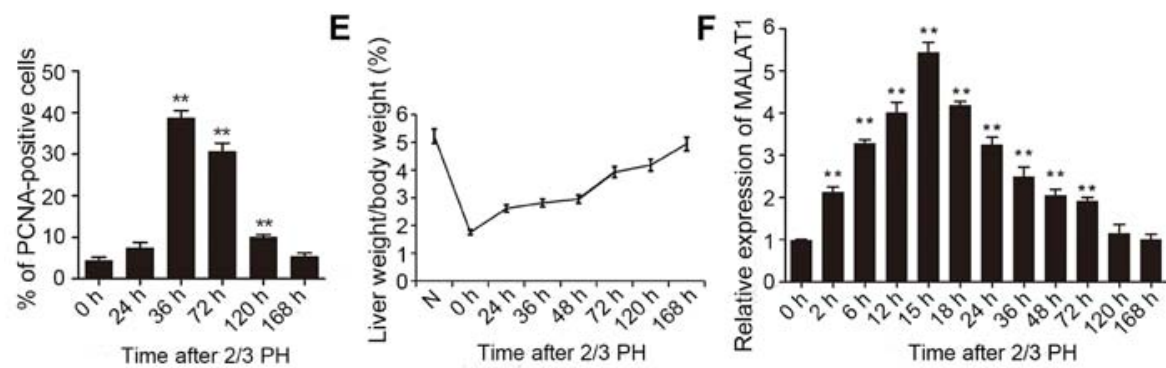

Figure 1. Metastasis-associated lung adenocarcinoma transcript 1 (MALAT1) participates in liver regeneration following 2/3 partial hepatectomy (PH). Mice were subjected to $2 / 3 \mathrm{PH}$. (A-C) The expression of alanine aminotransferase (ALT), aspartate aminotransferase (AST) and albumin (ALB) was detected at different time points following hepatectomy by enzyme-linked immunosorbent assay (ELISA). (D) Hematoxylin and eosin (H\&E) staining revealed a normally regenerating mouse liver section at different time-points after $2 / 3 \mathrm{PH}$. The black arrows indicate the cells in mitosis (scale bars, $30 \mu \mathrm{m}$ ). Immunohistochemistry for proliferating cell nuclear antigen (PCNA) showing the differences in hepatocyte proliferation at 15,36,72 and $120 \mathrm{~h}$ following $2 / 3 \mathrm{PH}$ (scale bars, $30 \mu \mathrm{m}$ ). (E) The residual liver/body weight ratio was calculated at various time points. (F) The expression of MALAT1 at different time points was determined by RT-qPCR. The data are presented as the means \pm SD of 3 independent experiments. ${ }^{*} \mathrm{p}<0.05,{ }^{* *} \mathrm{p}<0.01 \mathrm{vs}$. the $0 \mathrm{~h}$ time point..

HGF increases MALAT1 expression. As MALAT1 expression increased during the early stages after $2 / 3 \mathrm{PH}$ and HGF rapidly increases following $\mathrm{PH}$ and plays a substantial role during liver regeneration (6), we hypothesized that the increase in MALAT1 expression may be mediated by HGF. To confirm our hypothesis, the NCTC 1469 cells were treated with 5, 10 or $20 \mathrm{ng} / \mathrm{ml} \mathrm{HGF}$ to induce hepatocyte proliferation. As shown in Fig. 3A, MALAT1 expression gradually increased following treatment with increasing concentrations of HGF. The increased expression of HGF was detected in the BALB/c mice subjected to $2 / 3 \mathrm{PH}$ (Fig. 3B). Moreover, as shown in Fig. 3C, we identified a correlation between the HGF and MALAT1 levels in the liver tissue at different time points following 2/3 $\mathrm{PH}$.

MALAT1 activates Wnt/ $\beta$-catenin signaling by inhibiting Axinl and adenomatous polyposis coli (APC) in vitro. It has been demonstrated that the $\mathrm{Wnt} / \beta$-catenin pathway is associated with proliferation and differentiation (21). Thus, to determine whether MALAT1 accelerates hepatocyte proliferation by activating the $\mathrm{Wnt} / \beta$-catenin pathway, we evaluated the levels of cyclin D1, $\beta$-catenin, the $\beta$-catenin-degradation component, Axin1, glycogen synthase kinase (GSK)-3 $\beta$ and APC in MALAT1-silenced BNL CL.2 cells and
MALAT1-overexpressing NCTC 1469 cells. The results revealed an increase in the protein and $m R N A$ levels of $\beta$-catenin and cyclin D1 in the MALAT1-overexpressing NCTC 1469 cells (Fig. 4A, C and E). The protein and mRNA levels of Axin1 and APC decreased when MALAT1 was overexpressed in the NCTC 1469 cells (Fig. 4B and C); however, there was no significant change in the level of GSK-3 $\beta$ (Fig. 4B and C). The level of c-Myc increased when MALAT1 was overexpressed, but declined following the knockdown of MALAT1 (Fig. 4C).

As shown in Fig. 4D and E, inactive $\beta$-catenin was located in the cytoplasm, and total $\beta$-catenin staining decreased when MALAT1 was silenced in the BNL CL.2 cells. In addition, western blot analysis of $\beta$-catenin demonstrated that the level of $\beta$-catenin in the nucleus also decreased significantly when MALAT1 was silenced. These effects were reversed when MALAT1 was overexpressed. There was no significant difference in the level of cytoplasmic $\beta$-catenin when MALAT1 was silenced or overexpressed (Fig. 4E). $\beta$-catenin phosphorylation occurs in a multiprotein complex that includes the GSK-3 $\beta$, $\beta$-catenin, Axin 1 and APC proteins; this phosphorylation leads to the degradation of cytoplasmic $\beta$-catenin $(22,23)$.

On the whole, our data demonstrate that MALAT1 promotes cell cycle progression and accelerates hepatocyte proliferation during liver regeneration by activating $\mathrm{Wnt} / \beta$-catenin signaling. 

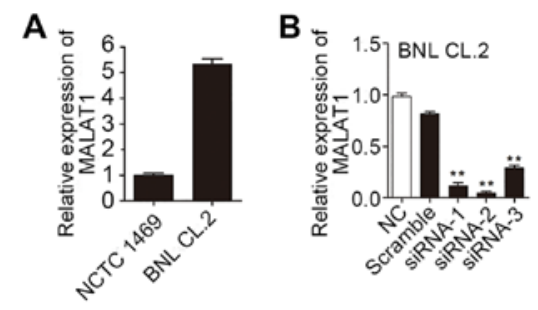

D

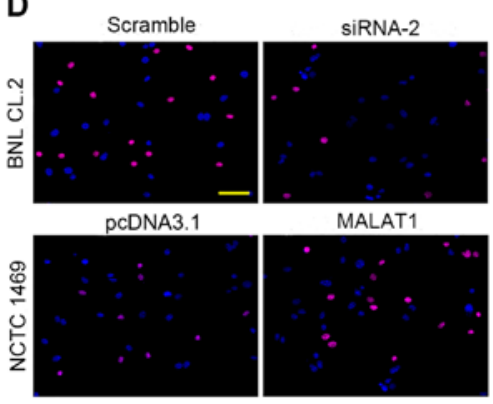

$\mathbf{F}$
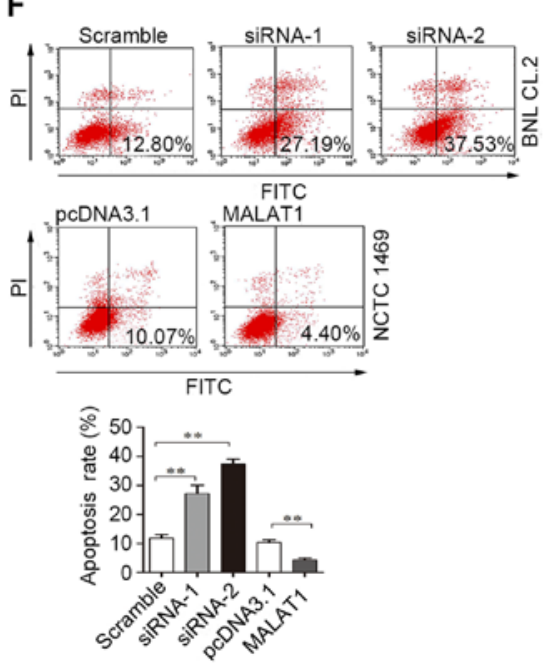
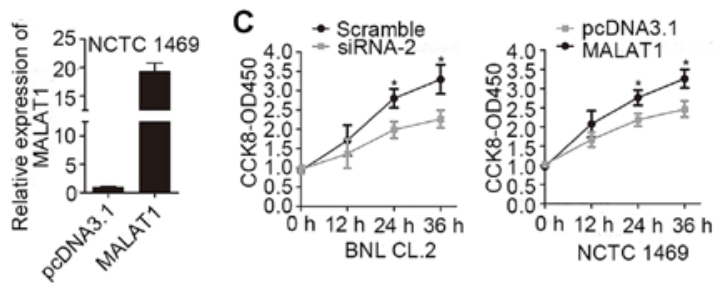

\section{E}
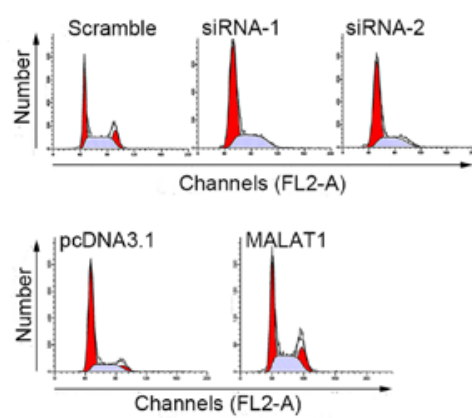

G
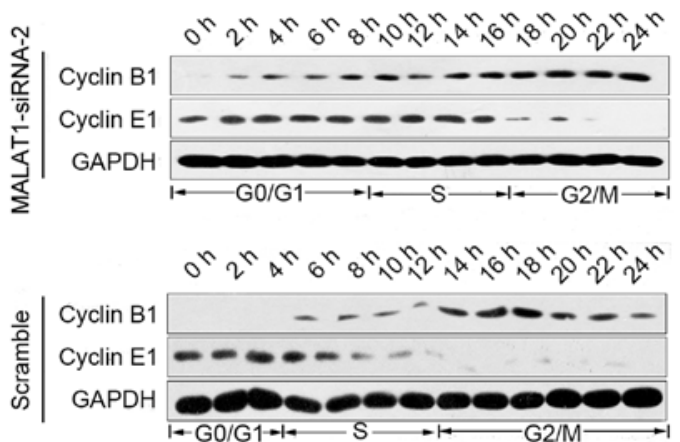

Figure 2. Metastasis-associated lung adenocarcinoma transcript 1 (MALAT1) accelerates the hepatocyte cell cycle and inhibits apoptosis in vitro. (A) The expression of MALAT1 in BNL CL.2 cells and NCTC 1469 cells was determined by RT-qPCR. The level of MALAT1 in NCTC 1469 cells was set to a value of 1. (B) RT-qPCR analysis of MALAT1 expression after MALAT1 was knocked down by siRNA in BNL CL.2 cells. MALAT1 expression was determined by RT-qPCR after MALAT1 was overexpressed in NCTC 1469 cells. (C) Cell proliferation was assessed by CCK-8 assay. (D) Cell proliferation was assessed using 5-ethynyl-2'-deoxyuridine (EdU) immunofluorescence staining. (E) FACS analysis showing that the number of cells in the G1/G0 phase increased significantly in MALAT1-silenced BNL CL.2 cells and that the number of cells in the S phase increased in MALAT1-overexpressing NCTC 1469 cells. (F) FACS analysis showing a significant increase in the number of apoptotic cells when MALAT1 was silenced in BNL CL.2 cells and a decrease in the number of apoptotic NCTC 1469 cells following transfection with pcDNA3.1-MALAT1. The percentage of apoptotic cells is also shown. (G) Western blot analysis of the expression of cyclin B1 and E1 in MALAT1-silenced BNL CL.2 cells at different time points during the cell cycle. The data are presented as the means \pm SD of 3 independent experiments. ${ }^{*} \mathrm{p}<0.05,{ }^{* *} \mathrm{p}<0.01 \mathrm{vs}$ the control or the $0 \mathrm{~h}$ time point.
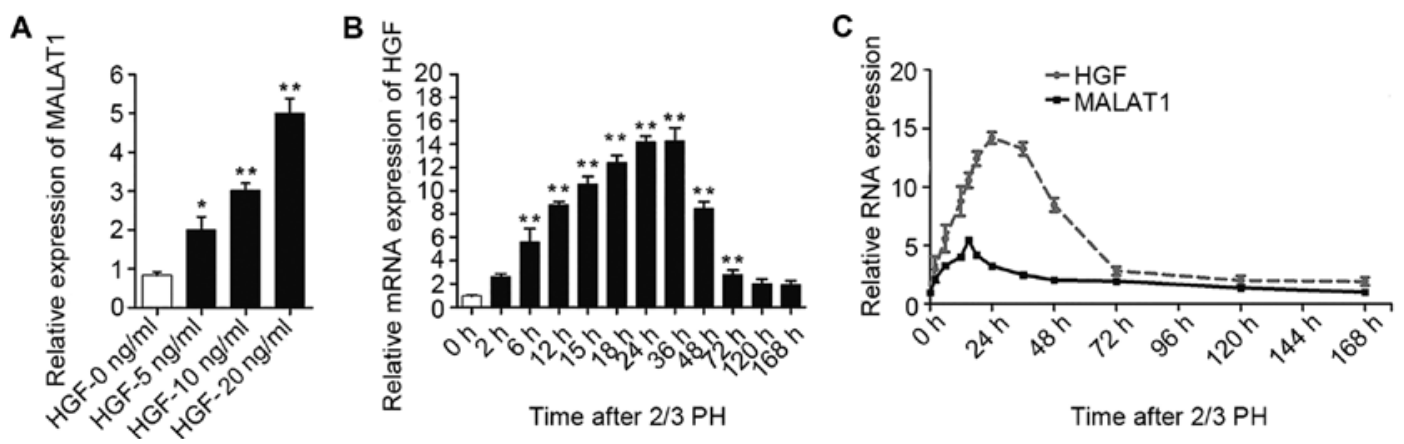

Figure 3. Hepatocyte growth factor (HGF) stimulates the expression of long non-coding RNA (lncRNA)-metastasis-associated lung adenocarcinoma transcript 1 (MALAT1). (A) The expression of MALAT1 increased in NCTC 1469 cells following treatment with increasing HGF concentrations. (B) Relative mRNA expression of HGF at different time points following 2/3 partial hepatectomy (PH). (C) The expression of MALAT1 and HGF in mouse liver samples was detected by RT-qPCR at various time points following $2 / 3 \mathrm{PH}$. The data are presented as the means $\pm \mathrm{SD}$ of 3 independent experiments. ${ }^{*} \mathrm{p}<0.05,{ }^{* *} \mathrm{p}<0.01$ vs. the control (no treatment) or the $0 \mathrm{~h}$ time point. 




Figure 4. Metastasis-associated lung adenocarcinoma transcript 1 (MALAT1) activates the Wnt/ $\beta$-catenin pathway by suppressing Axin1 and APC. (A) RT-qPCR analysis revealing the levels of $\beta$-catenin and cyclin D1 in MALAT1-silenced BNL CL.2 cells and MALAT1-overexpressing NCTC 1469 cells. (B) Changes in the expression of the $\beta$-catenin destruction complex (Axin1, APC and GSK-3 $\beta$ ) after the silencing or the overexpression of MALAT1 were detected by RT-qPCR. (C) Changes in the expression of the $\beta$-catenin destruction complex (Axin1, APC and GSK-3 $\beta$ ) and downstream target genes (cyclin D1 and c-Myc) after the silencing or overexpression of MALAT1 were detected by western blot analysis. The value below each band indicates the fold change in the protein level. (D) Changes in the expression and distribution of $\beta$-catenin in the cytoplasm and nucleus were observed by immunofluorescence (IF) staining after the silencing or overexpression of MALAT1. Yellow arrows indicate the translocation of $\beta$-catenin (scale bars, $20 \mu \mathrm{m}$ ). (E) The levels of nuclear, cytoplasmic and total $\beta$-catenin protein after the silencing or overexpression of MALAT1 are shown. The data are presented as the mean \pm SD of 3 independent experiments. ${ }^{*} \mathrm{p}<0.05,{ }^{* *} \mathrm{p}<0.01$ vs. the respective control.

By suppressing Axin1 and APC, MALAT1 indirectly reduces the stability of the $\beta$-catenin degradation complex, decreasing the level of inactive $\beta$-catenin, while increasing the level of active $\beta$-catenin. Active $\beta$-catenin is released and begins functioning. Subsequently, active $\beta$-catenin translocates to the nucleus to controls the transcription of target genes, such as c-Myc and cyclin D1. Finally, MALAT1 enhances hepatocyte proliferation by promoting cell cycle progression in vitro.

p53 regulates MALAT1 expression during liver regeneration. p53 activation is involved in hepatocyte proliferation following PH (24-26). A previous demonstrated shown that the downregulation of MALAT1 or the upregulation of p53 suppresses the proliferation of murine hematopoietic cells, as p53 specifically binds to the core promoter region of MALAT1. The core binding sequence is CGGCATGGCCGCCAAGGTCGCCGT GCCCT (27).

In this study, in order to determine the biological relevance of the p53-mediated regulation of MALAT1 in vivo, we examined the mRNA and protein levels of p53 during liver regeneration. The results of IHC and western blot analysis indicated that 2/3 PH decreased p53 expression in BALB/c mice, with p53 expression being detectable at $6 \mathrm{~h}$, with the lowest levels being observed at $15 \mathrm{~h}$, and the highest at $120 \mathrm{~h}$. The levels of p53 at 72 and $168 \mathrm{~h}$ were close to the levels observed $0 \mathrm{~h}$ following
PH (Fig. 5A and B). A correlation between MALAT1 and p53 may exist during liver regeneration (Fig. 5C). Cellular functional analysis indicated that MALAT1 had an effect on hepatocyte apoptosis, and thus we specifically detected apoptosis at $15 \mathrm{~h}$ following $2 / 3 \mathrm{PH}$. The proportion of cells undergoing apoptosis was $11 \%$ at $15 \mathrm{~h}$, and the level of apoptosis may be related to p53 and MALAT1 (Fig. 5D). Thus, we hypothesized that MALAT1 may be negatively regulated by p53 during liver regeneration.

\section{Discussion}

The key finding of the present study was that MALAT1 is upregulated during mouse liver regeneration. MALAT1 may acts as a regulatory factor in the cell cycle. Our results also demonstrated that both HGF and p53 are closely associated with MALAT1 during liver regeneration. MALAT1 can activate $\mathrm{Wnt} / \beta$-catenin signaling in vitro. Axin1 and APC were suppressed by MALAT1, and the stability of the $\beta$-catenin degradation complex became weak. Active $\beta$-catenin translocated from the cytoplasm to the nucleus, where it then enhanced the transcription of c-Myc and cyclin D1. Ultimately, MALAT1 facilitated hepatocyte proliferation by promoting cell cycle progression from the G1 to the S phase (Fig. 6).

By binding to the promoter of MALAT1, p53 can negatively regulate the expression of MALAT1 $(17,27)$. MALAT1 is 
A

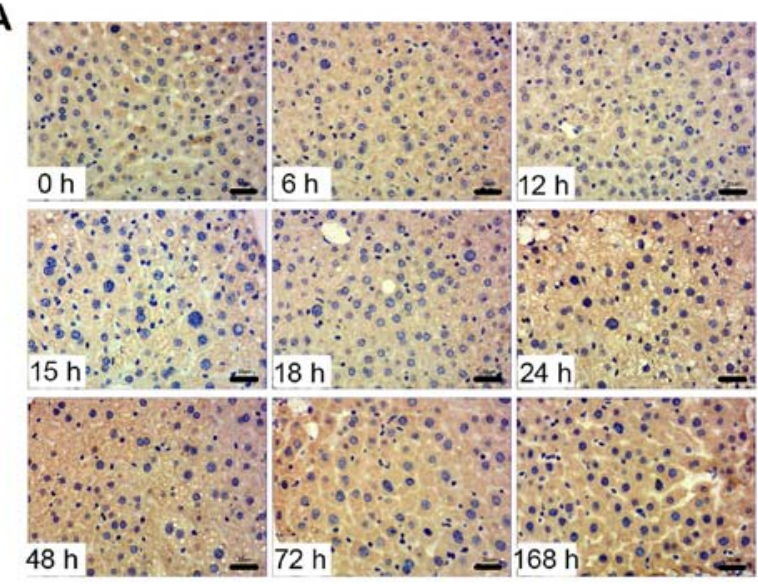

B

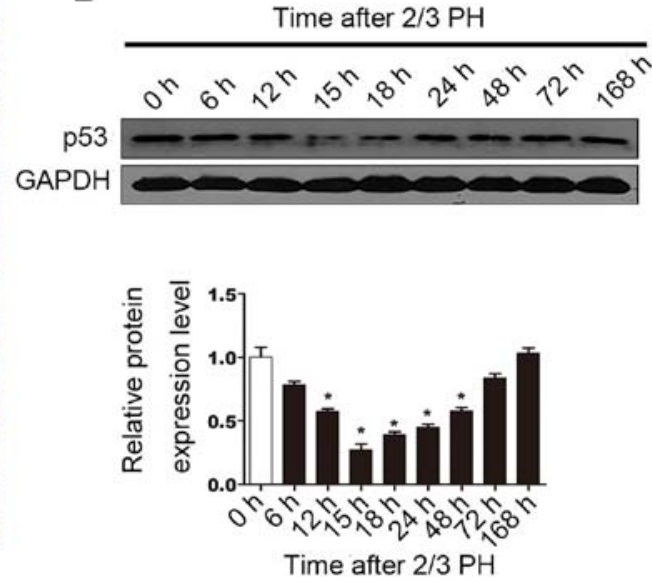

C

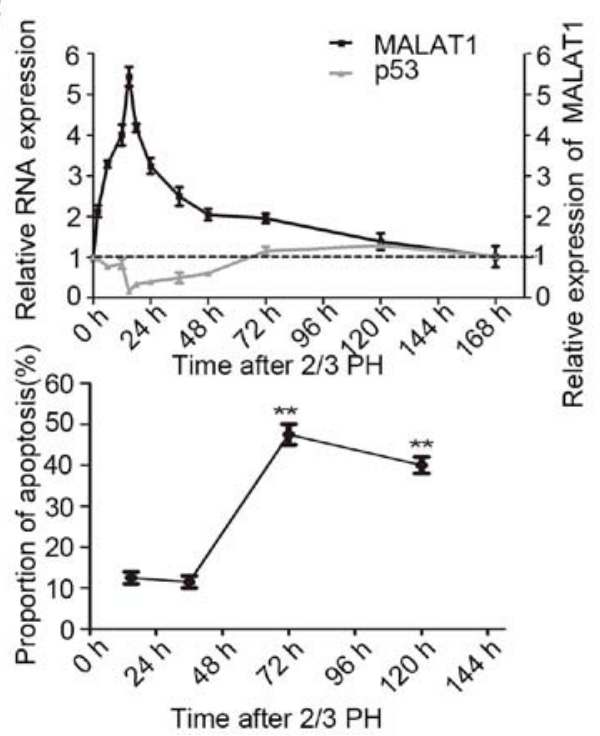

D

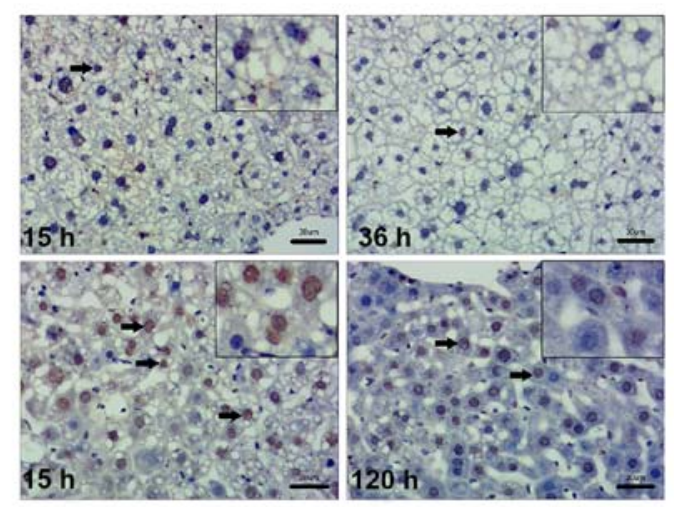

Figure 5. p53 protein negatively regulates metastasis-associated lung adenocarcinoma transcript 1 (MALAT1) in hepatocytes. (A) Representative images showing the p53 protein levels detected by immunohistochemistry (IHC) at different time points following $2 / 3$ partial hepatectomy (PH) (scale bars, $30 \mu \mathrm{m}$ ). (B) Western blot analysis of the changes in p53 protein expression at different time points following $2 / 3 \mathrm{PH}$. The graph in the bottom panel shows the percentage gray value in each band relative to the control. (C) The expression of p53 and MALAT1 in mouse liver samples was detected by RT-qPCR following 2/3 PH. (D) The proportion of cells undergoing apoptosis following $2 / 3 \mathrm{PH}$. All the results were reproduced in at least 3 independent experiments. The data are presented as the means $\pm \mathrm{SD}$ of 3 independent experiments. ${ }^{*} \mathrm{p}<0.05,{ }^{* *} \mathrm{p}<0.01 \mathrm{vs}$. the $0 \mathrm{~h}$ time point. The boxes (insets) on the top right of the images indicate local amplification in order to let the reader see more clearly. The arrows in the images indicate apoptosis cells.

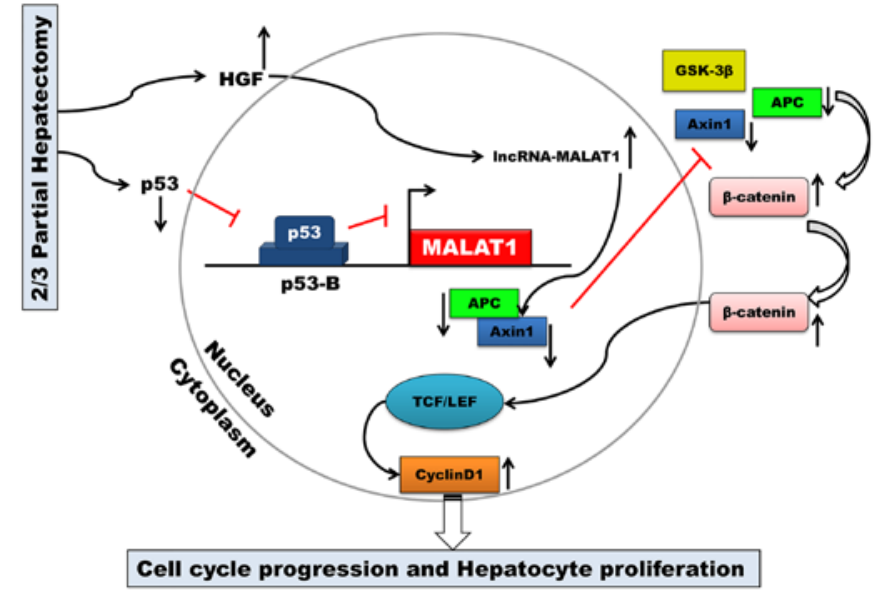

Figure 6. Schematic representation of the biological role of long noncoding RNA (lncRNA)-metastasis-associated lung adenocarcinoma transcript 1 (MALAT1) and mechanisms of IncRNA MALAT1-mediated liver regeneration. an essential factor in maintaining the proliferative potential of early-stage hepatocytes. Combined with the results of our study, these results indicate that MALAT1 may be transcriptionally regulated by $\mathrm{p} 53$ during liver regeneration following 2/3 $\mathrm{PH}$.

Although a number of cytokines, growth factors (such as HGF) and miRNAs regulate proliferation-related genes during liver regeneration $(28,29)$, the role of lncRNAs in liver regeneration remains largely unknown. A comprehensive analysis of IncRNA expression during mouse liver regeneration has revealed changes in the expression of multiple lncRNAs (14). In this study, we found that HGF increased MALAT1 expression in vitro; however, the peak in HGF expression was delayed compared to the peak in MALAT1 expression. The abovementioned phenomenon indicates that HGF may function as a regulatory factor for MALAT1. Between 15 and $24 \mathrm{~h}$ following $\mathrm{PH}$, the anti-MALAT1 effects override the HGF-mediated increase in MALAT1, indicating that additional signaling molecules and transcription factors may regulate the expression 
of MALAT1; we aim to continue to investigate the mechanisms underlying the association between HGF and MALAT1 in future studies. The HGF and Wnt/ $\beta$-catenin pathways both play key roles in accelerating hepatocyte proliferation and can cooperate to stimulate hepatocyte proliferation, which may be crucial in liver regeneration following PH (30).

The complete liver regeneration process takes approximately 7 days in mice; however, the mechanisms through which the liver initiates and terminates regenerative growth are not yet fully understood (31). Exploring the biological functions of lncRNAs has recently come to the foreground $(12,32)$. Previous studies investigating the non-coding RNAs in the liver have mainly focused on the association between the presence of these RNAs and the development of hepatocellular carcinoma (HCC) or the differential expression of these RNAs in carcinomas and the adjacent tissue (33). Some genes associated with hepatocyte proliferation and liver regeneration are also expressed abnormally in HCC $(34,35)$. Liver regeneration is a specific cellular process that is both similar to and different from HCC (36). To date, it remains unclear as to whether the lncRNAs regulating cell proliferation in $\mathrm{HCC}$ participate in liver regeneration. The overall mechanisms warrant further investigation. Although partial liver transplantation can minimize organ scarcity, the risk of death of a healthy donor, the development of SFSS in both the donor and recipient and technical complexity limit its applicability. Compounds that interfere with the signaling pathways that regulate proliferation are receiving increased attention and may be used to prevent or reverse SFSS (37). In conclusion, to the best of our knowledge, our research group is the first to report a biological role of MALAT1 in hepatocyte proliferation. MALAT1 is expected to become an indicator of prognosis for a variety of liver diseases and liver resection.

\section{References}

1. Dutkowski P, Linecker M, DeOliveira ML, Müllhaupt B and Clavien PA: Challenges to liver transplantation and strategies to improve outcomes. Gastroenterology 148: 307-323, 2015.

2. Lehmann K, Tschuor C, Rickenbacher A, Jang JH, Oberkofler CE, Tschopp O, Schultze SM, Raptis DA, Weber A, Graf R, et al: Liver failure after extended hepatectomy in mice is mediated by a p21-dependent barrier to liver regeneration. Gastroenterology 143: 1609-1619, 2012.

3. Petrowsky H, Breitenstein S, Slankamenac K, Vetter D, Lehmann K, Heinrich S, DeOliveira ML, Jochum W, Weishaupt D, Frauenfelder T, et al: Effects of pentoxifylline on liver regeneration: a double-blinded, randomized, controlled trial in 101 patients undergoing major liver resection. Ann Surg 252: 813-822, 2010.

4. Michalopoulos GK: Liver regeneration after partial hepatectomy: critical analysis of mechanistic dilemmas. Am J Pathol 176: 2-13, 2010.

5. Selzner N, Selzner M, Tian Y, Kadry Z and Clavien PA: Cold ischemia decreases liver regeneration after partial liver transplantation in the rat: a TNF-alpha/IL-6-dependent mechanism. Hepatology 36: 812-818, 2002.

6. Pediaditakis P, Lopez-Talavera JC, Petersen B, Monga SP and Michalopoulos GK: The processing and utilization of hepatocyte growth factor/scatter factor following partial hepatectomy in the rat. Hepatology 34: 688-693, 2001.

7. Fausto N, Campbell JS and Riehle KJ: Liver regeneration. Hepatology 43 (Suppl 1): S45-S53, 2006.

8. Shu J, Kren BT, Xia Z, Wong PY, Li L, Hanse EA, Min MX, Li B, Albrecht JH, Zeng Y, et al: Genomewide microRNA downregulation as a negative feedback mechanism in the early phases of liver regeneration. Hepatology 54: 609-619, 2011.

9. Song G, Sharma AD, Roll GR, Ng R, Lee AY, Blelloch RH, Frandsen NM and Willenbring H: MicroRNAs control hepatocyte proliferation during liver regeneration. Hepatology 51: 1735-1743, 2010.
10. Castro RE, Ferreira DM, Zhang X, Borralho PM, Sarver AL, Zeng Y, Steer CJ, Kren BT and Rodrigues CM: Identification of microRNAs during rat liver regeneration after partial hepatectomy and modulation by ursodeoxycholic acid. Am J Physiol Gastrointest Liver Physiol 299: G887-G897, 2010.

11. Forbes SJ and Newsome PN: Liver regeneration - mechanisms and models to clinical application. Nat Rev Gastroenterol Hepatol 13: 473-485, 2016.

12. Dey BK, Mueller AC and Dutta A: Long non-coding RNAs as emerging regulators of differentiation, development, and disease. Transcription 5: e944014, 2014.

13. Ørom UA, Derrien T, Beringer M, Gumireddy K, Gardini A, Bussotti G, Lai F, Zytnicki M, Notredame C, Huang Q, et al: Long noncoding RNAs with enhancer-like function in human cells. Cell 143: 46-58, 2010.

14. Xu D, Yang F, Yuan JH, Zhang L, Bi HS, Zhou CC, Liu F, Wang F and Sun SH: Long noncoding RNAs associated with liver regeneration 1 accelerates hepatocyte proliferation during liver regeneration by activating $\mathrm{Wnt} / \beta$-catenin signaling. Hepatology 58: 739-751, 2013.

15. Yamamoto $Y$, Nishikawa $Y$, Tokairin $T$, Omori $Y$ and Enomoto $K$ : Increased expression of H19 non-coding mRNA follows hepatocyte proliferation in the rat and mouse. J Hepatol 40: 808-814, 2004

16. Spector DL and Lamond AI: Nuclear speckles. Cold Spring Harb Perspect Biol 3: pii: a000646, 2011.

17. Tripathi V, Shen Z, Chakraborty A, Giri S, Freier SM, Wu X, Zhang Y, Gorospe M, Prasanth SG, Lal A and Prasanth KV: Long noncoding RNA MALAT1 controls cell cycle progression by regulating the expression of oncogenic transcription factor B-MYB. PLoS Genet 9: e1003368, 2013.

18. Mitchell C and Willenbring H: A reproducible and well-tolerated method for $2 / 3$ partial hepatectomy in mice. Nat Protoc 3 : $1167-1170,2008$

19. Bockhorn M, Goralski M, Prokofiev D, Dammann P, Grünewald P, Trippler M, Biglarnia A, Kamler M, Niehues EM, Frilling A, et al: VEGF is important for early liver regeneration after partial hepatectomy. J Surg Res 138: 291-299, 2007.

20. Chang L, Li C, Guo T, Wang H, Ma W, Yuan Y, Liu Q, Ye Q and Liu Z: The human RNA surveillance factor UPF1 regulates tumorigenesis by targeting Smad7 in hepatocellular carcinoma. J Exp Clin Cancer Res 35: 8, 2016.

21. Tan X, Behari J, Cieply B, Michalopoulos GK and Monga SP: Conditional deletion of beta-catenin reveals its role in liver growth and regeneration. Gastroenterology 131: 1561-1572, 2006.

22. Li VS, Ng SS, Boersema PJ, Low TY, Karthaus WR, Gerlach JP, Mohammed S, Heck AJ, Maurice MM, Mahmoudi T and Clevers H: Wnt signaling through inhibition of $\beta$-catenin degradation in an intact Axinl complex. Cell 149: 1245-1256, 2012.

23. Ha NC, Tonozuka T, Stamos JL, Choi HJ and Weis WI: Mechanism of phosphorylation-dependent binding of APC to beta-catenin and its role in beta-catenin degradation. Mol Cell 15: 511-521, 2004.

24. Zhang L, Liu L, He Z, Li G, Liu J, Song Z, Jin H, Rudolph KL, Yang $\mathrm{H}$, Mao Y, et al: Inhibition of wild-type p53-induced phosphatase 1 promotes liver regeneration in mice by direct activation of mammalian target of rapamycin. Hepatology 61: 2030-2041, 2015.

25. Chen S, Zheng J, Hao Q, Yang S, Wang J, Chen H, Chen L, Zhou Y, Yu C, Jiao B and Cai Z: p53-insensitive PUMA down-regulation is essential in the early phase of liver regeneration after partial hepatectomy in mice. J Hepatol 52: 864-871, 2010.

26. Stepniak E, Ricci R, Eferl R, Sumara G, Sumara I, Rath M, Hui L and Wagner EF: c-Jun/AP-1 controls liver regeneration by repressing p53/p21 and p38 MAPK activity. Genes Dev 20: 2306-2314, 2006.

27. Ma XY, Wang JH, Wang JL, Ma CX, Wang XC and Liu FS: Malat1 as an evolutionarily conserved lncRNA, plays a positive role in regulating proliferation and maintaining undifferentiated status of early-stage hematopoietic cells. BMC Genomics 16 : 676, 2015.

28. Ng R, Song G, Roll GR, Frandsen NM and Willenbring H: A microRNA-21 surge facilitates rapid cyclin D1 translation and cell cycle progression in mouse liver regeneration. J Clin Invest 122: 1097-1108, 2012.

29. Yuan Q, Loya K, Rani B, Möbus S, Balakrishnan A, Lamle J, Cathomen T, Vogel A, Manns MP, Ott M, et al: MicroRNA-221 overexpression accelerates hepatocyte proliferation during liver regeneration. Hepatology 57: 299-310, 2013. 
30. Apte U, Zeng G, Muller P, Tan X, Micsenyi A, Cieply B, Dai C, Liu Y,Kaestner KH and Monga SP: Activation of Wnt/beta-catenin pathway during hepatocyte growth factor-induced hepatomegaly in mice. Hepatology 44: 992-1002, 2006.

31. Cox AG and Goessling W: Regenerative biology: take the brakes off for liver repair. Nature 506: 299-300, 2014

32. Meola N, Pizzo M, Alfano G, Surace EM and Banfi S: The long noncoding RNA Vax2os 1 controls the cell cycle progression of photoreceptor progenitors in the mouse retina. RNA 18: 111-123, 2012.

33. He Y, Meng XM, Huang C, Wu BM, Zhang L, Lv XW and Li J: Long noncoding RNAs: novel insights into hepatocelluar carcinoma. Cancer Lett 344: 20-27, 2014

34. Bandiera S, Pfeffer S, Baumert TF and Zeisel MB: miR-122 - a key factor and therapeutic target in liver disease. J Hepatol 62: 448-457, 2015
35. John K, Hadem J, Krech T, Wahl K, Manns MP, Dooley S, Batkai S, Thum T, Schulze-Osthoff K and Bantel H: MicroRNAs play a role in spontaneous recovery from acute liver failure. Hepatology 60: 1346-1355, 2014.

36. Gandhi CR, Chaillet JR, Nalesnik MA, Kumar S, Dangi A, Demetris AJ, Ferrell R, Wu T, Divanovic S, Stankeiwicz T, et al: Liver-specific deletion of augmenter of liver regeneration accelerates development of steatohepatitis and hepatocellular carcinoma in mice. Gastroenterology 148: 379-391, 2015.

37. Huang W, Ma K, Zhang J, Qatanani M, Cuvillier J, Liu J, Dong B, Huang X and Moore DD: Nuclear receptor-dependent bile acid signaling is required for normal liver regeneration. Science 312: 233-236, 2006. 